\title{
Context is Key: Grammatical Error Detection with Contextual Word Representations
}

\author{
Samuel Bell ${ }^{\diamond}$ Helen Yannakoudakis ${ }^{\diamond}$ Marek Rei ${ }^{\diamond \bullet}$ \\ -Department of Psychology, University of Cambridge, United Kingdom \\ $\diamond^{\diamond}$ Dept. of Computer Science \& Technology, University of Cambridge, United Kingdom \\ *ALTA Institute, University of Cambridge, United Kingdom \\ sjb326@cam.ac.uk, \{helen.yannakoudakis, marek.rei\}@cl.cam.ac.uk
}

\begin{abstract}
Grammatical error detection (GED) in nonnative writing requires systems to identify a wide range of errors in text written by language learners. Error detection as a purely supervised task can be challenging, as GED datasets are limited in size and the label distributions are highly imbalanced. Contextualized word representations offer a possible solution, as they can efficiently capture compositional information in language and can be optimized on large amounts of unsupervised data. In this paper, we perform a systematic comparison of ELMo, BERT and Flair embeddings (Peters et al., 2017; Devlin et al., 2018; Akbik et al., 2018) on a range of public GED datasets, and propose an approach to effectively integrate such representations in current methods, achieving a new state of the art on GED. We further analyze the strengths and weaknesses of different contextual embeddings for the task at hand, and present detailed analyses of their impact on different types of errors.
\end{abstract}

\section{Introduction}

Detecting errors in text written by language learners is a key component of pedagogical applications for language learning and assessment. Supervised learning approaches to the task exploit public error-annotated corpora (Yannakoudakis et al., 2011; Ng et al., 2014; Napoles et al., 2017) that are, however, limited in size, in addition to having a biased distribution of labels: the number of correct tokens in a text far outweighs the incorrect (Leacock et al., 2014). As such, Grammatical Error Detection (GED) can be considered a low/mid-resource task.

The current state of the art explores error detection within a semi-supervised, multi-task learning framework, using a neural sequence labeler optimized to detect errors as well as predict their surrounding context (Rei, 2017). To further improve GED performance, recent work has investigated the use of artificially generated training data (Rei et al., 2017; Kasewa et al., 2018). On the related task of grammatical error correction (GEC), Junczys-Dowmunt et al. (2018) explore transfer learning approaches to tackle the low-resource bottleneck of the task and, among others, find substantially improved performance when incorporating pre-trained word embeddings (Mikolov et al., 2013), and importing network weights from a language model trained on a large unlabeled corpus.

Herein, we extend the current state of the art for error detection (Rei, 2017) to effectively incorporate contextual embeddings: word representations that are constructed based on the context in which the words appear. These embeddings are typically the output of a set of hidden layers of a large language modelling network, trained on large volumes of unlabeled and general domain data. As such, they are able to capture detailed information regarding language and composition from a wide range of data sources, and can help overcome resource limitations for supervised learning.

We evaluate the use of contextual embeddings in the form of Bidirectional Encoder Representations from Transformers (BERT) (Devlin et al., 2018), embeddings from Language Models (ELMo) (Peters et al., 2018) and Flair embeddings (Akbik et al., 2018). To the best of our knowledge, this is the first evaluation of the use of contextual embeddings for the task of GED. Our contributions are fourfold:

- We present a systematic comparison of different contextualized word representations for the task of GED;

- We describe an approach for effectively integrating contextual representations to error detection models, achieving a new state of the 
art on a number of public GED datasets, and make our code and models publicly available online;

- We demonstrate that our approach has particular benefits for transferring to out-of-domain datasets, in addition to overall improvements in performance;

- We perform a detailed analysis of the strengths and weaknesses of different contextual representations for the task of GED, presenting detailed results of their impact on different types of errors in order to guide future work.

\section{Related work}

In this section, we describe previous work on GED and on the related task of GEC. While error correction systems can be used for error detection, previous work has shown that standalone error detection models can be complementary to error correction ones, and can be used to further improve performance on GEC (Yannakoudakis et al., 2017).

Early approaches to GED and GEC relied upon handwritten rules and error grammars (e.g. Foster and Vogel (2004)), while later work focused on supervised learning from error-annotated corpora using feature engineering approaches and often utilizing maximum entropy-based classifiers (e.g. Chodorow et al. (2007); De Felice and Pulman (2008)). A large range of work has focused on the development of systems targeting specific error types, such as preposition (Tetreault and Chodorow, 2008; Chodorow et al., 2007), article usage (Han et al., 2004, 2006), and verb form errors (Lee and Seneff, 2008). Among others, errortype agnostic approaches have focused on generating synthetic ungrammatical data to augment the available training sets, or learning from native English datasets; for example, Foster and Andersen (2009) investigate rule-based error generation methods, while Gamon (2010) trains a language model (LM) on a large, general domain corpus, from which features (e.g. word likelihoods) are derived for use in error classification.

As a distinct task, GEC has been formulated as a naïve-bayes classification (Rozovskaya et al., 2013, 2014; Rozovskaya and Roth, 2016) or a monolingual (statistical or neural) machine translation (MT) problem (where uncorrected text is treated as the source "language" and the corrected text as its target counterpart) (Felice et al., 2014; Junczys-Dowmunt and Grundkiewicz, 2014; Rozovskaya and Roth, 2016; Yuan and Briscoe, 2016).

Recently, Rei and Yannakoudakis (2016) presented the first approach towards neural GED, training a sequence labeling model based on word embeddings processed by a bidirectional LSTM (bi-LSTM), outputting a probability distribution over labels informed by the entire sentence as context. This approach achieves strong results when trained and evaluated on in-domain data, but shows weaker generalization performance on outof-domain data. Rei et al. (2016) extended this model to include character embeddings in order to capture morphological similarities such as word endings. Rei (2017) subsequently added a secondary LM objective to the neural sequence labeling architecture, operating on both word and character-level embeddings. This was found to be particularly useful for GED - introducing an LM objective allows the network to learn more generic features about language and composition. At the same time, Rei and Yannakoudakis (2017) investigated the effectiveness of a number of auxiliary (morpho-syntactic) training objectives for the task of GED, finding that predicting part-ofspeech tags, grammatical relations or error types as auxiliary tasks yields improvements in performance over the single-task GED objective (though not as high as when utilizing an LM objective).

The current state of the art on GED is based on augmenting neural approaches with artificially generated training data. Rei et al. (2017) showed improved GED performance using the bi-LSTM sequence labeler, by generating artificial errors in two different ways: 1) learning frequent error patterns from error-annotated corpora and applying these to error-free text; 2) using a statistical MT approach to "translate" correct text to its incorrect counterpart using parallel corpora. Recently, Kasewa et al. (2018) applied the latter approach using a neural MT system instead, and achieved a new state of the art on GED using the neural model of Rei (2017).

\section{Data}

In this section, we describe the different public datasets we use to train our models.

The First Certificate in English (FCE) dataset 
(Yannakoudakis et al., 2011) is a publicly-released set of essays written by non-native learners of English taking a language assessment exam. Each essay is annotated by professional annotators with the spans of language errors committed, the types of errors, and suggested corrections. In addition, the CoNLL 2014 shared task on GEC (Ng et al., 2014) used a dataset of English essays written by advanced undergraduate students at the National University of Singapore. Each essay is annotated by two experienced annotators and has error annotations similarly to the FCE, though using a different error taxonomy. The Johns Hopkins University (JHU) FLuency-Extended GUG Corpus (JFLEG) dataset (Napoles et al., 2017) contains essays written by a range of English learners with different first languages and proficiency levels. Each essay is corrected by four annotators with native-level proficiency and annotated with fluency and grammar edits.

The 2019 Building Educational Applications (BEA) shared task on GEC (Bryant et al., 2019) released two new datasets: the Cambridge English Write \& Improve (W\&I) corpus, which is a collection of texts written by learners of English of varying levels of proficiency and submitted for assessment to the Write \& Improve system (Yannakoudakis et al., 2018), an automated online tool for writing feedback; and the LOCNESS corpus (Granger, 1998), originally compiled at the Centre for English Corpus Linguistics at the University of Louvain, and comprising essays written by native English students. Both datasets are annotated for corrections by the W\&I annotators.

In this work, we use the FCE training set as training data, and evaluate our models on the FCE test set, the CoNLL-2014 test set, the JFLEG test set, and the BEA 2019 shared task development and test sets. This setup allows us to investigate the extent to which our models and the use of contextualized representations transfer to out-ofdomain data.

We follow Rei and Yannakoudakis (2016) and convert the span-based annotations in these datasets to binary error detection labels at the token level (i.e. is a token correct or incorrect). Performance is evaluated using precision, recall, and $F_{0.5}$ at the token level. $F_{0.5}$ places twice the weight on precision than recall: systems that incorrectly penalize correct language can have a much more negative impact to language learning compared to systems that miss to detect some errors (Ng et al., 2014). We note that performance on the BEA shared task test set is conducted using the official evaluation tool in CodaLab.

We also perform detailed analyses in order to evaluate the performance of our models per error type. As the datasets above either have their own error type taxonomy or they are not annotated with error types at all, we follow the 2019 BEA shared task and induce error types for all datasets automatically using the ERRor ANnotation Toolkit (ERRANT) (Bryant et al., 2017). ERRANT automatically annotates parallel uncorrected and corrected sentences with error types using a universal error taxonomy and hence allowing for comparisons across datasets. The system uses distancebased alignment followed by rule-based error categorization. An error type is hence assigned to every incorrect token in each dataset, with the exception of the BEA 2019 shared task test set, for which the corrected versions are not yet publicly available.

\section{Error detection model}

In this section, we extend the current state of the art (neural) architecture for GED (Rei, 2017), which we use as our baseline system. This model is a bi-LSTM sequence labeler over token embeddings where, for each token, the model is trained to output a probability distribution over binary correct/incorrect labels using a softmax layer (i.e. predicting whether a token is correct or incorrect in context). The model is additionally trained with a secondary bidirectional LM objective, predicting the surrounding context of the target token in the sequence. Specifically, the model uses a forward LM to predict the next token in the sequence, and a backward LM to predict the previous token. Rei (2017) also makes use of a character-level biLSTM, as opposed to solely conditioning on tokens, in order to benefit from sub-word morphological units, of particular use in the case of unknown or incorrectly spelled words. The outputs of the character-level LSTMs are concatenated to the word embeddings and given as input to the word-level bi-LSTM.

The model learns 300-dimensional word embeddings, initialized with pre-trained Google News embeddings (Mikolov et al., 2013), ${ }^{1}$ and

\footnotetext{
${ }^{1}$ https: //code.google.com/archive/p/ word2vec/
} 


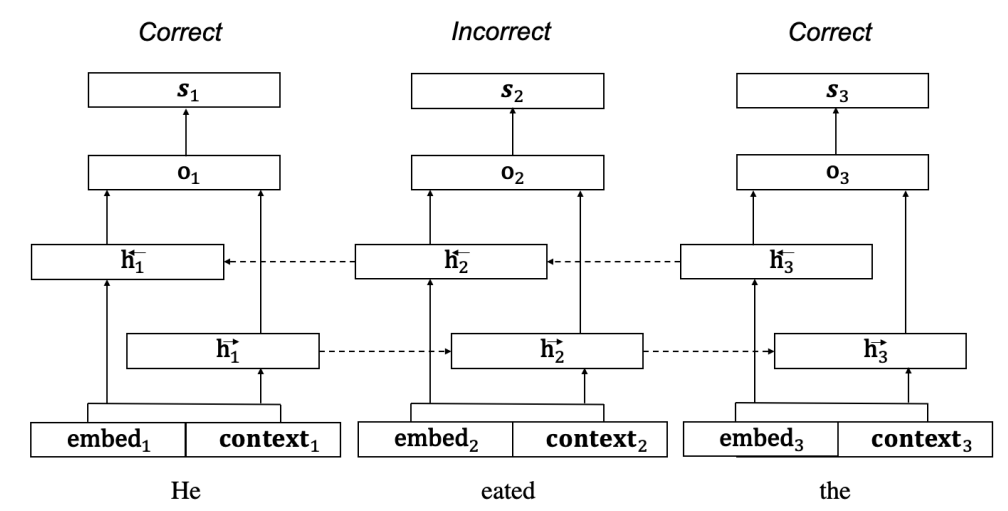

(a)

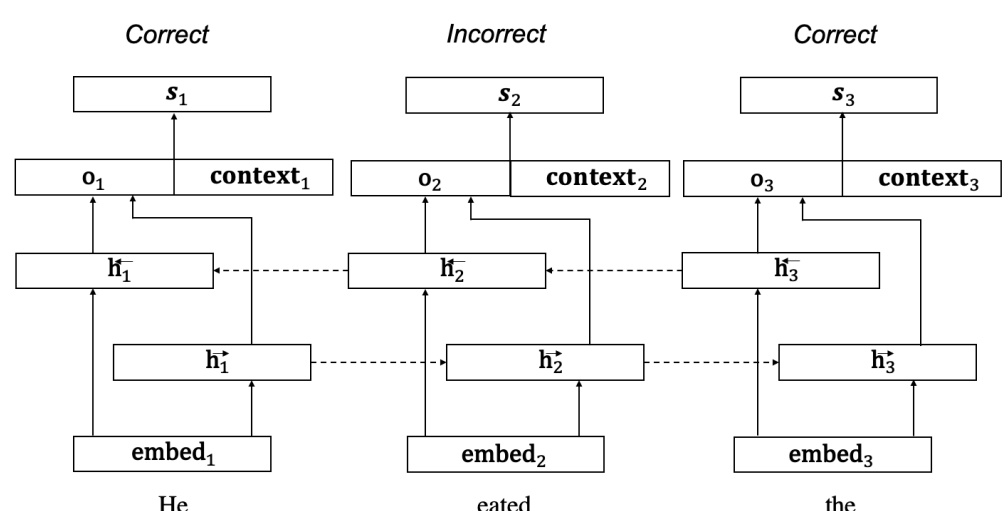

(b)

Figure 1: Simplified bi-LSTM sequence labeler with: (a) contextual embeddings (context) concatenated to the input word embeddings (embed), before being passed through the bi-LSTM (h); (b) contextual embeddings (context) concatenated to the LSTM output (o) before being passed through a softmax layer (s).

100-dimensional character embeddings. The hidden states of the word- and character-level LSTMs are also of 300 and 100 dimensions respectively. The outputs of each LSTM are passed through a 50-dimensional hidden layer with a tanh activation function. Dropout is applied to the inputs and outputs of each LSTM with a probability of 0.5. The model is trained with a crossentropy loss function for the error detection objective that minimizes the negative log probability of the gold label. As the model is also trained with a secondary LM objective, a second bipartite cross-entropy loss function is used, minimizing the negative log probability of the next word in the sequence for the forward LM, and the previous word for the backward LM. A hyperparameter $\gamma=0.1$ weights the combination of the two loss functions, assigning more importance to the main task of error detection over the auxiliary task of language modelling. Optimization is performed with the AdaDelta optimizer (Zeiler, 2012), using an initial learning rate of 1.0 , and batches of 32 sentences. Training is terminated when validation performance does not improve for 7 epochs.

In this work, we extend the above model by incorporating contextualized word embeddings, produced by three different approaches (BERT, ELMo and Flair; each described in more detail in Section 5). Specifically, we concatenate the contextual embeddings either to the input word embeddings before being passed through the wordlevel bi-LSTM (Figure 1a), or to the bi-LSTM's output (Figure 1b). Peters et al. (2018) find that the best point to integrate ELMo vectors varies by task and, as such, we continue that line of analysis here.

We make a TensorFlow (Abadi et al., 2016) implementation of our code and models publicly 
available online. $^{2}$

\section{Contextualized embeddings}

Three types of contextual embeddings are considered in this work: BERT, ELMo and Flair embeddings (Peters et al., 2017; Devlin et al., 2018; Akbik et al., 2018). In each case, we use the publiclyavailable pre-trained models released by the authors.

BERT embeddings are extracted from the highest layers of a transformer architecture trained with a masked LM objective: rather than predicting the next or previous word in a sequence, a percentage of input tokens are masked and then the network learns to predict the masked tokens. BERT is also trained with a second objective predicting whether one sentence directly follows another, given two input sentences. BERT pretrained embeddings are available in two variants: base embeddings, which are the concatenation of the four highest 768-dimension hidden layers, yielding a 3,072-dimension embedding; large embeddings, which are the concatenation of the four highest 1024-dimension hidden layers, yielding a 4, 096-dimension embedding (Devlin et al., 2018). BERT embeddings are trained on the BooksCorpus ( 0.8 billion words) of books written by unpublished authors (Zhu et al., 2015) and English Wikipedia ( 2.5 billion words).

ELMo embeddings are a weighted elementwise sum of the outputs of three-layered stacked bi-LSTM LMs, trained to predict both the next and previous token in the sequence. Using a taskspecific scalar per layer, the outputs of the three LSTMs are reduced to a single 1,024-dimension embedding (Peters et al., 2018). This task-specific weighting is learned by our sequence labeler during training. ELMo is trained on the One Billion Word Benchmark corpus ( 0.8 billion words), composed primarily of online news articles (Chelba et al., 2014a).

Flair embeddings are the concatenated output of a single (i.e. unstacked) character-level bi-LSTM. We use the concatenation of both the 2,048dimension "news-forward" and "news-backward" embeddings, each produced by a forward and backward bi-LSTM respectively, and both trained on the One Billion Word Benchmark (Chelba et al., 2014b). This yields a 4,096-dimensional

\footnotetext{
2https://github.com/samuel jamesbell/ sequence-labeler
}

embedding (Akbik et al., 2018).

\section{Results}

Table 1 and Table 2 present the results of integrating different contextual embeddings with the current state-of-the-art model described by Rei (2017). ${ }^{3}$ The experiments in this section are based on models with contextual representations concatenated to the word embeddings; Section 6.1 includes a more detailed investigation of different integration points. For comparison, we also report the results of Rei et al. (2017) and Kasewa et al. (2018), who improve error detection performance by additionally augmenting Rei (2017)'s model with artificial training data.

The experiments demonstrate a substantial improvement in precision, recall and $F_{0.5}$ for every model incorporating contextual embeddings, across every dataset considered. On the FCE test set, even our lowest performing model (Flair, $\left.F_{0.5}=49.97\right)$ outperforms the baseline $\left(F_{0.5}=\right.$ $42.15)$, with a relative improvement of $18.55 \%$. Our best performing model (BERT base, $F_{0.5}=$ 57.28) outperforms the baseline by a relative $35.9 \%$. This is also the new state-of-the-art result on the FCE test set, without using additional manually-annotated training data.

The best performance on the CoNLL-2014 test set is achieved by BERT large $\left(F_{0.5}=36.94\right)$ and BERT base $\left(F_{0.5}=46.29\right)$ for the first and second annotator respectively. These scores show more than $30 \%$ relative improvement over the previous best results by Kasewa et al. (2018), even without using additional artificial training data, for both annotators. On the JFLEG test set (Table 2), and both the BEA 2019 GEC Shared Task development and test sets, BERT base yields the highest performance. The improvement on the BEA shared task datasets is particularly large, with BERT base achieving $69.70 \%$ relative improvement on the development set and $63.30 \%$ relative improvement on the test set, compared to the baseline model.

These experiments demonstrate that contextual embeddings provide a very beneficial addition for GED systems, achieving a new state of the art across all datasets. Learning to compose language

\footnotetext{
${ }^{3}$ We include the results reported by Rei (2017) along with our re-trained baseline. We note that the differences in performance are due to a re-processing of the data in order to align parallel original-corrected sentences and derive fine-grained error type labels for later analyses (see Section 6.2).
} 


\begin{tabular}{lrrrrrrrrr}
\hline & \multicolumn{3}{c}{ CoNLL Test 1 } & \multicolumn{3}{c}{ CoNLL Test 2 } & \multicolumn{3}{c}{ FCE test } \\
& $P$ & $R$ & $F_{0.5}$ & $P$ & $R$ & $F_{0.5}$ & $P$ & $R$ & $F_{0.5}$ \\
\hline Rei (2017) & 17.68 & 19.07 & 17.86 & 27.6 & 21.18 & 25.88 & 58.88 & 28.92 & 48.48 \\
Rei et al. (2017) & 23.28 & 18.01 & 21.87 & 35.28 & 19.42 & 30.13 & 60.67 & 28.08 & 49.11 \\
Kasewa et al. (2018) & - & - & 28.3 & - & - & 35.5 & - & - & 55.6 \\
\hline Baseline & 20.82 & 16.31 & 19.73 & 31.91 & 17.81 & 27.55 & 46.55 & 30.58 & 42.15 \\
\hline Flair & 29.53 & 17.11 & 25.79 & 44.12 & 18.22 & 34.35 & 58.36 & 31.72 & 49.97 \\
ELMo & 30.83 & 23.90 & 29.14 & 46.66 & 25.77 & 40.15 & 58.50 & 38.01 & 52.81 \\
BERT base & 37.62 & 29.65 & 35.70 & $\mathbf{5 3 . 5 2}$ & 30.05 & $\mathbf{4 6 . 2 9}$ & 64.96 & $\mathbf{3 8 . 8 9}$ & $\mathbf{5 7 . 2 8}$ \\
BERT large & $\mathbf{3 8 . 0 4}$ & $\mathbf{3 3 . 1 2}$ & $\mathbf{3 6 . 9 4}$ & 51.40 & $\mathbf{3 1 . 8 9}$ & 45.80 & $\mathbf{6 4 . 5 1}$ & 38.79 & 56.96 \\
\hline
\end{tabular}

Table 1: Error detection precision, recall, and $F_{0.5}$ on the FCE and CoNLL-2014 test sets: test 1 and test 2 refer to the two different CoNLL annotators. 'Baseline' refers to our own re-training of the model by Rei (2017).

\begin{tabular}{lrrrrrrrrr}
\hline & \multicolumn{3}{c}{ JFLEG Test } & \multicolumn{3}{c}{ Shared Task Dev } & \multicolumn{3}{c}{ Shared Task Test } \\
& $P$ & $R$ & $F_{0.5}$ & $P$ & $R$ & $F_{0.5}$ & $P$ & $R$ & $F_{0.5}$ \\
\hline Baseline & 72.84 & 22.83 & 50.65 & 31.31 & 21.18 & 28.58 & 40.05 & 34.99 & 38.93 \\
\hline Flair & 75.65 & 25.26 & 54.08 & 41.80 & 24.10 & 36.45 & 53.40 & 39.84 & 50.00 \\
ELMo & 74.95 & 31.21 & 58.54 & 47.90 & 30.41 & 42.96 & 58.72 & 47.79 & 56.15 \\
BERT base & $\mathbf{7 9 . 5 1}$ & 32.94 & $\mathbf{6 1 . 9 8}$ & $\mathbf{5 3 . 3 1}$ & 35.65 & $\mathbf{4 8 . 5 0}$ & $\mathbf{6 6 . 4 7}$ & $\mathbf{5 4 . 1 1}$ & $\mathbf{6 3 . 5 7}$ \\
BERT large & 76.47 & $\mathbf{3 4 . 5 2}$ & 61.52 & 51.54 & $\mathbf{3 6 . 9 0}$ & 47.75 & 63.35 & 54.10 & 61.26 \\
\hline
\end{tabular}

Table 2: Error detection precision, recall, and $F_{0.5}$ on the JFLEG test set and BEA 2019 GEC Shared Task development and test sets.

representations on large unsupervised datasets allows the models to access a wider range of useful information. While our error detection models are optimized on the FCE training set, we observe particularly large improvements on the CoNLL2014 and BEA shared task datasets, indicating that contextual embeddings allow the models to generalize better and capture errors in out-of-domain texts. Overall, we found BERT base to provide the highest improvements across all datasets. The slightly lower performance of BERT large could be attributed to the larger embedding sizes requiring more parameters to be optimized on our limited GED dataset.

\subsection{Integration method}

We performed additional experiments to investigate the optimal method for integrating contextual embeddings into the error detection model. The embeddings are either concatenated to the standard word embeddings at the input level (reported in our results as 'input'), or to the output of the word-level bi-LSTM (reported as 'output'). In all experiments, contextual embeddings are not finetuned.

Table 3 compares the $F_{0.5}$ of these two strategies for each model, across all the datasets. We observe that, although performance varies across datasets and models, integration by concatena- tion to the word embeddings yields the best results across the majority of datasets for all models (BERT: 3/5 datasets; ELMo: 4/5 datasets; Flair: $5 / 5$ datasets). The lower integration point allows the model to learn more levels of task-specific transformations on top of the contextual representations, leading to an overall better performance.

\subsection{Error type performance}

Using ERRANT (Bryant et al., 2017), we analyze the performance on different types of errors and specifically focus on two error type taxonomies: one that uses part-of-speech (POS) based error types (i.e. the type is based on the POS tag of the incorrect token), and another based on edit operations (i.e. is it a missing token, an unnecessary token, or a replace token error). This allows us to yield insights into how different types of contextual embeddings and the data in which they were trained may impact performance on specific errors. Since identifying type-specific false positives is not possible in this setting, we follow $\mathrm{Ng}$ et al. (2014) and report recall on each error type.

Figure 2 presents the performance on POSbased error types, showing the change in error type recall of each contextual embedding model compared to our baseline, averaged over all datasets. While all models yield an improvement in aggregate performance metrics $\left(P, R, F_{0.5}\right)$, when bro- 


\begin{tabular}{llrrrrr}
\hline & & Shared Task Dev & CoNLL Test 1 & CoNLL Test 2 & FCE test & JFLEG test \\
\hline \multirow{2}{*}{ Flair } & Input & 36.45 & 25.79 & 34.35 & 49.97 & 54.08 \\
& Output & 33.47 & 24.52 & 33.18 & 48.50 & 52.10 \\
\hline \multirow{2}{*}{ ELMo } & Input & 42.96 & 29.14 & 40.15 & 52.81 & 58.54 \\
& Output & 37.33 & 27.33 & 38.10 & 52.99 & 54.86 \\
\hline \multirow{2}{*}{ BERT base } & Input & $\mathbf{4 8 . 5 0}$ & 35.70 & 46.29 & $\mathbf{5 7 . 2 8}$ & $\mathbf{6 1 . 9 8}$ \\
& Output & 46.33 & 37.04 & 46.50 & 55.32 & 60.97 \\
\hline \multirow{2}{*}{ BERT large } & Input & 47.75 & 36.94 & 45.80 & 56.96 & 61.52 \\
& Output & 46.72 & $\mathbf{3 9 . 0 7}$ & $\mathbf{4 6 . 9 6}$ & 55.10 & 60.56 \\
\hline
\end{tabular}

Table 3: Error detection $F_{0.5}$ of different embedding integration strategies ('input' vs. 'output') per model on all datasets.

ken down by POS-based error type, some trends emerge. BERT base, BERT large and ELMo each show strong improvements in recall of errors relating to nouns, particles, prepositions and morphology. In comparison, relatively weak improvements are achieved for errors of conjugation, orthography and spelling. As such words/errors are less likely to occur frequently in general-purpose corpora of English (i.e. spelling mistakes are less frequent in news articles compared to learner essays), contextual embeddings trained on such corpora are also less helpful for these error types.

We also note the sharp decline in recall of the BERT base model on contraction errors. This error type occurs quite infrequently (see Figure 1 in the Appendix) and we do not observe this issue with BERT large.

Compared to BERT and ELMo, Flair offers very little improvement on POS-based error type recall or even actively degrades recall (e.g. conjugation, punctuation or spelling errors). While the purely character-based representations of Flair could potentially offer more flexibility in the model, these results suggest that the limited vocabulary of our learner data may be better captured with word-level approaches.

Figure 3 presents the differences between models when looking at error types based on the necessary edit operation: missing token, replace token or unnecessary token. While BERT improves overall performance compared to ELMo, this improvement appears to be limited to replacement and unnecessary error types. On missing word errors, BERT base performs comparably to ELMo and BERT large even decreases performance. We discuss the possible reasons for this in Section 7.

We include the full results table for different error types in the Appendix (Table 1, Table 2). Our analysis shows that focusing more on punctu- ation, determiner and preposition errors might be the most beneficial avenue for improving the overall performance on GED. For example, punctuation errors are the third most common error type, but even with contextual embeddings the models only achieve $27.7 \%$ recall across all datasets.

Overall, our results suggest that, while contextual embeddings always improve aggregate performance on GED, error type specific properties of models should also be considered.

\section{Discussion}

The previous section has demonstrated consistent improvement in precision, recall and $F_{0.5}$ across a range of GED datasets, including many examples of transfer to different domains, irrespective of the choice of contextual embedding. Contextual embeddings bring the possibility of leveraging information learned in an unsupervised manner from high volumes of unlabeled data, by large and complex models, trained for substantial periods of time. For these reasons, they are a particularly appropriate addition to a low-resource task such as GED. While each choice of contextual embedding yields improved performance, BERT base and BERT large consistently outperform ELMo and Flair embeddings. Here, we suggest that details of the BERT architecture and training process may be responsible for its specific performance profile.

One difference between contextual embedding models is the choice of training corpora. While both ELMo and Flair embeddings are trained using the One Billion Word Benchmark, BERT is trained on the BooksCorpus, and English Wikipedia. It is likely that the usage and distribution of English varies across these corpora, yielding different results on downstream tasks. We might expect a corpus of books to exhibit a greater 

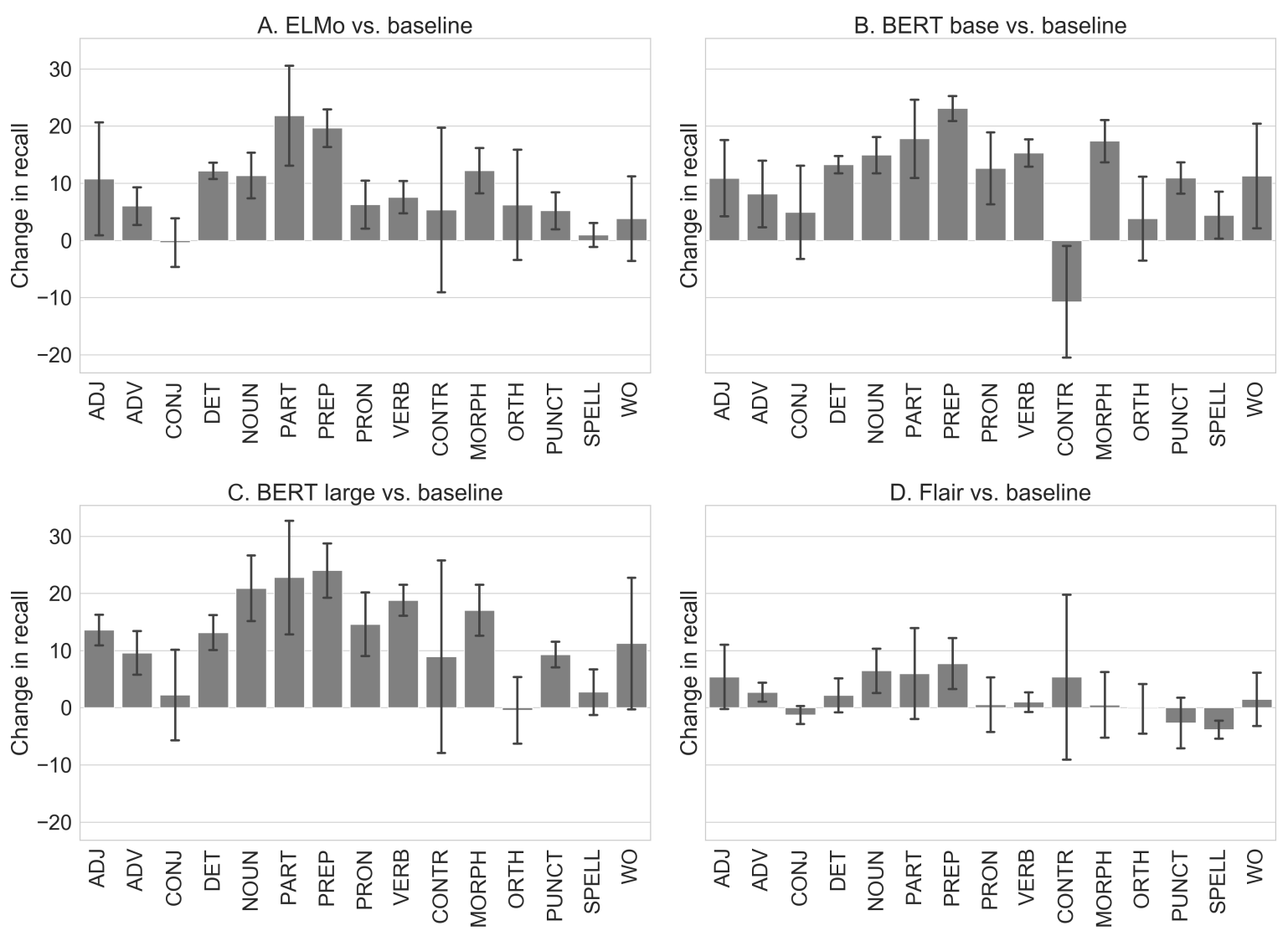

Figure 2: Mean change in recall of ERRANT-induced POS-based error types over all datasets when adding contextual embeddings at the input level, vs. our baseline without contextual embeddings. A: ELMo. B: BERT base. C: BERT large. D: Flair.

variance in writing style, audience, and even writer ability than a corpus of news articles, perhaps resulting in more useful contextual embeddings for GED. However, in contrast to the 0.8 billion tokens of training data available to ELMo and Flair, BERT's combination of BooksCorpus and English Wikipedia provides 3.3 billion tokens of training data. The increased volume of training data may alone suffice to explain BERT's comparatively strong performance.

Another difference is that BERT is not trained with a bi-directional LM objective. In contrast to ELMo and Flair, BERT is trained with a masked LM objective, such that it must predict the original tokens when presented with a sentence with any number of tokens masked. This training objective always provides the model access to the correct number of tokens in each sentence, which means it never needs to consider possible missing tokens. This could explain the decreased improvements on the "missing" error types compared to other error operations (Figure 3), and future work could ex- periment with integrating missing tokens directly into the BERT training objective.

At this point, we note that while the number of parameters, and the dimensionality of the resulting representations vary by model, extensive ablation studies (Devlin et al., 2018; Peters et al., 2018) indicate only small decreases in performance with decreasing layers or dimensionality. Future research may contrast the models considered here with those of a paired number of parameters but with randomly-initialized contextual embeddings. However, as contextual embeddings enable the integration of information captured via unsupervised learning on large general-purpose corpora, we expect that embeddings without this information (i.e. randomly-initialized) would not yield the degree of improvement detailed herein.

\section{Conclusion}

We have experimentally demonstrated that using contextual embeddings substantially improves GED performance, achieving a new state of the art 

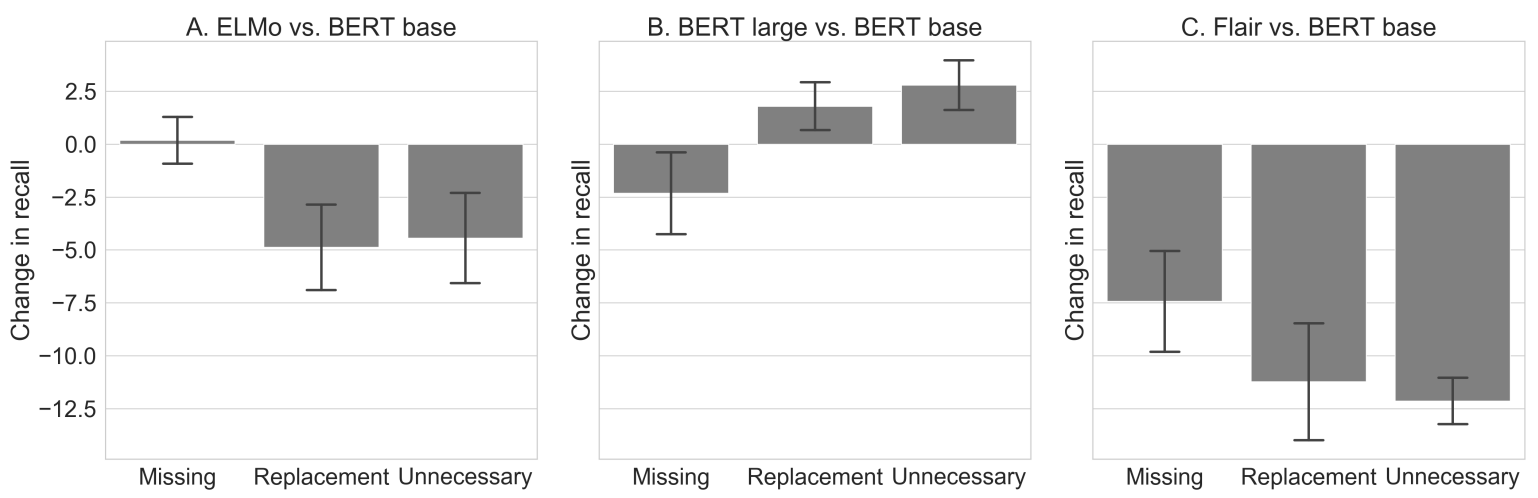

Figure 3: Mean change in recall of ERRANT-induced edit operation error types across all datasets when adding contextual embeddings at the input level, vs. the model using BERT base. A: ELMo. B: BERT large. C: Flair.

on a number of public datasets. We have shown that a sequence labeling architecture augmenting the input word embeddings with the BERT family (base or large) of contextual embeddings produces, overall, the best performance across datasets. In addition to improving overall performance, contextual embeddings proved to be particularly useful for improving on out-of-domain datasets.

We have also performed a detailed analysis of the strengths and weaknesses of the use of different contextual embeddings on detecting specific types of errors. We aim for the analyses presented here to facilitate future work in GED and in improving such systems further. Future work can also be directed towards investigating alternative approaches to integrating contextualized representations and fine-tuning such representations for GED.

\section{Acknowledgments}

We thank the anonymous reviewers for their valuable feedback. Marek Rei and Helen Yannakoudakis were supported by Cambridge Assessment, University of Cambridge.

\section{References}

Martín Abadi, Paul Barham, Jianmin Chen, Zhifeng Chen, Andy Davis, Jeffrey Dean, Matthieu Devin, Sanjay Ghemawat, Geoffrey Irving, Michael Isard, et al. 2016. Tensorflow: A system for large-scale machine learning. In 12th $\{$ USENIX $\}$ Symposium on Operating Systems Design and Implementation ( $\{O S D I\} 16)$, pages 265-283.

Alan Akbik, Duncan Blythe, and Vollgraf Roland. 2018. Contextual string embeddings for sequence labeling. In Proceedings of the 27th International Conference on Computational Linguistics, pages 1638-1649.

Christopher Bryant, Mariano Felice, Øistein E Andersen, and Ted Briscoe. 2019. The BEA-2019 shared task on grammatical error correction. In Proceedings of the 14th Workshop on Innovative Use of NLP for Building Educational Applications. Association for Computational Linguistics.

Christopher Bryant, Mariano Felice, and Ted Briscoe. 2017. Automatic annotation and evaluation of error types for grammatical error correction. In Proceedings of the 55th Annual Meeting of the Association for Computational Linguistics, pages 793-805.

Ciprian Chelba, Tomas Mikolov, Mike Schuster, Qi Ge, Thorsten Brants, Phillipp Koehn, and Tony Robinson. 2014a. One billion word benchmark for measuring progress in statistical language modeling. Proceedings of the Annual Conference of the International Speech Communication Association, INTERSPEECH, pages 2635-2639.

Ciprian Chelba, Tomáš Mikolov, Mike Schuster, Qi Ge, Thorsten Brants, Phillipp Koehn, and Tony Robinson. 2014b. One billion word benchmark for measuring progress in statistical language modeling. In Fifteenth Annual Conference of the International Speech Communication Association.

Martin Chodorow, Joel R Tetreault, and Na-Rae Han. 2007. Detection of grammatical errors involving prepositions. In Proceedings of the fourth ACLSIGSEM workshop on prepositions, pages 25-30.

Rachele De Felice and Stephen G. Pulman. 2008. A classifier-based approach to preposition and determiner error correction in L2 English. In Proceedings of the 22nd International Conference on Computational Linguistics (Coling 2008), pages 169176.

Jacob Devlin, Ming-Wei Chang, Kenton Lee, and Kristina Toutanova. 2018. BERT: Pre-training of 
deep bidirectional transformers for language understanding. arXiv preprint arXiv:1810.04805.

Mariano Felice, Zheng Yuan, Øistein E Andersen, Helen Yannakoudakis, and Ekaterina Kochmar. 2014. Grammatical error correction using hybrid systems and type filtering. In Proceedings of the Eighteenth Conference on Computational Natural Language Learning: Shared Task, pages 15-24.

Jennifer Foster and Øisten E Andersen. 2009. GenERRate: generating errors for use in grammatical error detection. In Proceedings of the fourth workshop on innovative use of NLP for building educational applications, pages 82-90.

Jennifer Foster and Carl Vogel. 2004. Parsing illformed text using an error grammar. Artificial Intelligence Review, 21(3-4):269-291.

Michael Gamon. 2010. Using mostly native data to correct errors in learners' writing: A meta-classifier approach. In Human Language Technologies: The 2010 Annual Conference of the North American Chapter of the Association for Computational Linguistics, pages 163-171. Association for Computational Linguistics.

Sylviane Granger. 1998. The computer learner corpus: A versatile new source of data for sla research. in granger, s. (ed.). In Learner English on Computer, pages 3-18. Addison Wesley Longman: London \& New York.

Na-Rae Han, Martin Chodorow, and Claudia Leacock. 2004. Detecting errors in English article usage with a maximum entropy classifier trained on a large, diverse corpus. In Proceedings of the Fourth International Conference on Language Resources and Evaluation (LREC'04).

Na-Rae Han, Martin Chodorow, and Claudia Leacock. 2006. Detecting errors in English article usage by non-native speakers. Natural Language Engineering, 12(2):115-129.

Marcin Junczys-Dowmunt and Roman Grundkiewicz. 2014. The AMU system in the CoNLL-2014 shared task: Grammatical error correction by dataintensive and feature-rich statistical machine translation. In Proceedings of the Eighteenth Conference on Computational Natural Language Learning: Shared Task, pages 25-33.

Marcin Junczys-Dowmunt, Roman Grundkiewicz, Shubha Guha, and Kenneth Heafield. 2018. Approaching neural grammatical error correction as a low-resource machine translation task. arXiv preprint arXiv:1804.05940.

Sudhanshu Kasewa, Pontus Stenetorp, and Sebastian Riedel. 2018. Wronging a right: Generating better errors to improve grammatical error detection. arXiv preprint arXiv: 1810.00668 .
Claudia Leacock, Martin Chodorow, Michael Gamon, and Joel Tetreault. 2014. Automated grammatical error detection for language learners. Morgan \& Claypool Publishers.

John Lee and Stephanie Seneff. 2008. Correcting misuse of verb forms. In Proceedings of the 46th Annual Meeting of the Association for Computational Linguistics: Human Language Technologies, pages 174-182.

Tomáš Mikolov, Kai Chen, Greg Corrado, and Jeffrey Dean. 2013. Efficient estimation of word representations in vector space. arXiv preprint arXiv:1301.3781.

Courtney Napoles, Keisuke Sakaguchi, and Joel Tetreault. 2017. JFLEG: A fluency corpus and benchmark for grammatical error correction. In Proceedings of the 15th Conference of the European Chapter of the Association for Computational Linguistics, pages 229-234.

Hwee Tou Ng, Siew Mei Wu, Ted Briscoe, Christian Hadiwinoto, Raymond Hendy Susanto, and Christopher Bryant. 2014. The CoNLL-2014 shared task on grammatical error correction. In Proceedings of the Eighteenth Conference on Computational Natural Language Learning: Shared Task, pages 1-14.

Matthew Peters, Waleed Ammar, Chandra Bhagavatula, and Russell Power. 2017. Semi-supervised sequence tagging with bidirectional language models. In Proceedings of the 55th Annual Meeting of the Association for Computational Linguistics, pages 1756-1765.

Matthew E. Peters, Mark Neumann, Mohit Iyyer, Matt Gardner, Christopher Clark, Kenton Lee, and Luke Zettlemoyer. 2018. Deep contextualized word representations. arXiv preprint arXiv:1802.05365.

Marek Rei. 2017. Semi-supervised multitask learning for sequence labeling. In Proceedings of the 55th Annual Meeting of the Association for Computational Linguistics, pages 2121-2130.

Marek Rei, Gamal Crichton, and Sampo Pyysalo. 2016. Attending to characters in neural sequence labeling models. In Proceedings of COLING 2016, the 26th International Conference on Computational Linguistics: Technical Papers, pages 309-318.

Marek Rei, Mariano Felice, Zheng Yuan, and Ted Briscoe. 2017. Artificial error generation with machine translation and syntactic patterns. arXiv preprint arXiv:1707.05236.

Marek Rei and Helen Yannakoudakis. 2016. Compositional sequence labeling models for error detection in learner writing. In Proceedings of the 54th Annual Meeting of the Association for Computational Linguistics, pages 1181-1191. 
Marek Rei and Helen Yannakoudakis. 2017. Auxiliary objectives for neural error detection models. In Proceedings of the 12th Workshop on Innovative Use of NLP for Building Educational Applications, pages 33-43. Association for Computational Linguistics.

Alla Rozovskaya, Kai-Wei Chang, Mark Sammons, and Dan Roth. 2013. The University of Illinois system in the CoNLL-2013 shared task. In Proceedings of the Seventeenth Conference on Computational Natural Language Learning: Shared Task, pages 13-19.

Alla Rozovskaya, Kai-Wei Chang, Mark Sammons, Dan Roth, and Nizar Habash. 2014. The IllinoisColumbia system in the CoNLL-2014 shared task. In Proceedings of the Eighteenth Conference on Computational Natural Language Learning: Shared Task, pages 34-42.

Alla Rozovskaya and Dan Roth. 2016. Grammatical error correction: Machine translation and classifiers. In Proceedings of the 54th Annual Meeting of the Association for Computational Linguistics, pages 2205-2215. Association for Computational Linguistics.

Joel R Tetreault and Martin Chodorow. 2008. The ups and downs of preposition error detection in ESL writing. In Proceedings of the 22nd International Conference on Computational Linguistics, pages 865-872.

Helen Yannakoudakis, Øistein E Andersen, Ardeshir Geranpayeh, Ted Briscoe, and Diane Nicholls. 2018. Developing an automated writing placement system for esl learners. Applied Measurement in Education, 31(3):251-267.

Helen Yannakoudakis, Ted Briscoe, and Ben Medlock. 2011. A new dataset and method for automatically grading ESOL texts. In Proceedings of the 49th Annual Meeting of the Association for Computational Linguistics: Human Language Technologies, pages 180-189.

Helen Yannakoudakis, Marek Rei, Øistein E Andersen, and Zheng Yuan. 2017. Neural sequence-labelling models for grammatical error correction. In Proceedings of the 2017 Conference on Empirical Methods in Natural Language Processing.

Zheng Yuan and Ted Briscoe. 2016. Grammatical error correction using neural machine translation. In Proceedings of the 2016 Conference of the North American Chapter of the Association for Computational Linguistics: Human Language Technologies, pages 380-386.

Matthew D Zeiler. 2012. ADADELTA: an adaptive learning rate method. arXiv preprint arXiv:1212.5701.

Yukun Zhu, Ryan Kiros, Rich Zemel, Ruslan Salakhutdinov, Raquel Urtasun, Antonio Torralba, and Sanja Fidler. 2015. Aligning books and movies: Towards story-like visual explanations by watching movies and reading books. In Proceedings of the IEEE international conference on computer vision, pages 19-27. 


\section{A Supplementary figures}

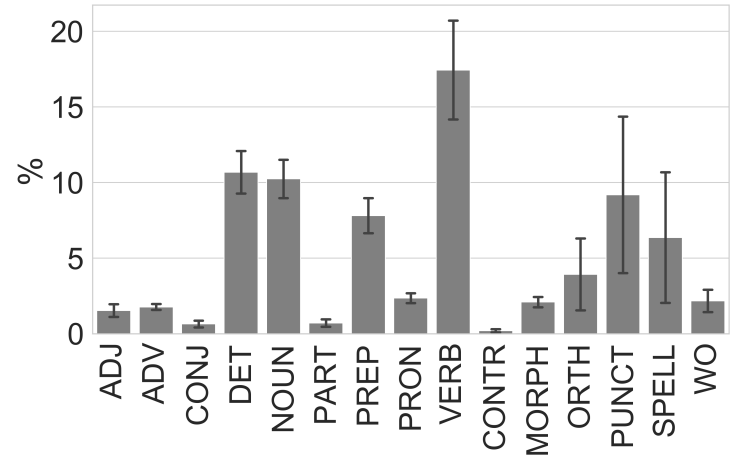

Figure 1: Mean proportion of ERRANT-induced POS-based error types across datasets (FCE test set, CoNLL 2014 test set 1 (both annotators), BEA 2019 GEC Shared Task development set and JFLEG test set). Error bars show the standard deviation.

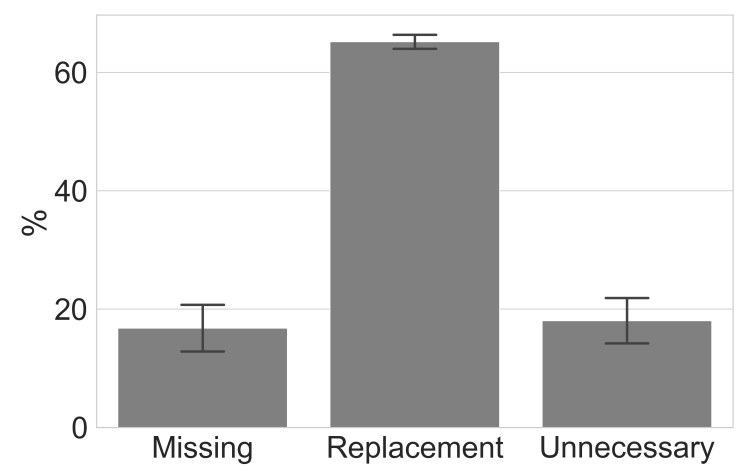

Figure 2: Mean proportion of ERRANT-induced edit operation error types across all datasets (FCE test set, CoNLL 2014 test set (both annotators), BEA 2019 GEC Shared Task development set and JFLEG test set). Error bars show the standard deviation. 


\section{B Supplementary tables}

\begin{tabular}{|c|c|c|c|c|c|c|}
\hline \multicolumn{7}{|c|}{$R$} \\
\hline & Baseline & BERT base & BERT large & ELMo & Flair & Frequency \\
\hline ADJ & 16.35 & 25.47 & 29.49 & 24.13 & 18.77 & 373 \\
\hline ADV & 8.18 & 16.62 & 17.39 & 13.81 & 11.25 & 391 \\
\hline CONJ & 9.63 & 15.56 & 12.59 & 8.89 & 8.15 & 135 \\
\hline CONTR & 18.60 & 11.63 & 20.93 & 25.58 & 25.58 & 43 \\
\hline DET & 24.78 & 38.11 & 38.02 & 37.28 & 27.30 & 2304 \\
\hline MORPH & 36.34 & 53.12 & 52.69 & 47.53 & 35.48 & 465 \\
\hline NOUN & 26.28 & 40.53 & 45.97 & 36.26 & 31.45 & 2245 \\
\hline ORTH & 29.40 & 28.96 & 28.20 & 30.49 & 27.76 & 915 \\
\hline OTHER & 18.77 & 29.96 & 31.06 & 27.04 & 20.69 & 4800 \\
\hline PART & 9.62 & 28.85 & 31.41 & 31.41 & 16.67 & 156 \\
\hline PREP & 10.70 & 34.71 & 35.25 & 31.40 & 20.42 & 1841 \\
\hline PRON & 12.20 & 25.98 & 28.35 & 19.69 & 13.78 & 508 \\
\hline PUNCT & 17.21 & 27.72 & 26.32 & 22.80 & 15.97 & 2504 \\
\hline SPELL & 88.33 & 92.88 & 91.48 & 89.06 & 84.88 & 1362 \\
\hline VERB & 18.63 & 33.75 & 36.80 & 25.40 & 19.32 & 3929 \\
\hline WO & 13.52 & 23.16 & 22.13 & 19.47 & 14.75 & 488 \\
\hline
\end{tabular}

Table 1: Overall recall of each model over all datasets broken out by ERRANT-induced POS-based error type, with frequency of occurrence of each error type.

\begin{tabular}{|c|c|c|c|c|c|c|}
\hline \multicolumn{7}{|c|}{$R$} \\
\hline & Baseline & BERT base & BERT large & ELMo & Flair & Frequency \\
\hline Missing & 19.00 & 28.98 & 26.83 & 29.17 & 22.48 & 3816 \\
\hline Replacement & 27.02 & 39.94 & 41.60 & 35.02 & 28.63 & 14839 \\
\hline Unnecessary & 15.38 & 29.07 & 31.81 & 24.50 & 16.72 & 3804 \\
\hline
\end{tabular}

Table 2: Overall recall of each model over all datasets broken out by ERRANT-induced edit operation error type, with frequency of occurrence of each error type. 